\title{
Risk Factors for Death Among Hospitalized Patients Aged 21-64 Years Diagnosed with COVID-19-New York City, March 13-April 9, 2020
}

\author{
Dena Bushman $^{1,2}$ (1) $\cdot$ Alexander Davidson ${ }^{1} \cdot$ Preeti Pathela $^{1} \cdot$ Sharon K. Greene ${ }^{1} \cdot$ Don Weiss ${ }^{1} \cdot$ Vasudha Reddy ${ }^{1}$. \\ New York City Fatal Case-Control Study Team ${ }^{1} \cdot$ Julia Latash $^{1}$
}

Received: 4 May 2021 / Revised: 14 June 2021 / Accepted: 28 June 2021 / Published online: 9 August 2021

(C) This is a U.S. government work and not under copyright protection in the U.S.; foreign copyright protection may apply 2021 , corrected publication 2021

\begin{abstract}
Background COVID-19 mortality studies have primarily focused on persons aged $\geq 65$ years; less is known about decedents aged $<65$ years.

Methods We conducted a case-control study among NYC residents aged 21-64 years hospitalized with COVID-19 diagnosed March 13-April 9, 2020, to determine risk factors for death. Case-patients ( $\mathrm{n}=343$ ) were hospitalized decedents with COVID-19 and control-patients ( $\mathrm{n}=686$ ) were discharged from hospitalization with COVID-19 and matched 2:1 to case-patients on age and residential neighborhood. Conditional logistic regression models were adjusted for patient sex, insurance status, and marital status. Matched adjusted odds ratios (aORs) were calculated for selected underlying conditions, combinations of conditions, and race/ethnic group.

Results Median age of both case-patients and control-patients was 56 years (range: $23-64$ years). Having $\geq 1$ selected underlying condition increased odds of death 4.45-fold (95\% CI: 2.33-8.49). Patients with diabetes; morbid obesity; heart, kidney, or lung disease; cancer; neurologic/neurodevelopmental conditions; mental health conditions; or HIV had significantly increased odds of death. Compared with having neither condition, having both diabetes and obesity or diabetes and heart disease was associated with approximately threefold odds of death. Five select underlying conditions were more prevalent among non-Hispanic Black control-patients than among control-patients of other races/ethnicities.

Conclusions and Relevance Selected underlying conditions were risk factors for death, and most prevalent among racial/ethnic minorities. Social services; health care resources, including vaccination; and tailored public health messaging are important for COVID-19 prevention. Strengthening these strategies for racial/ethnic minority groups could minimize COVID-19 racial/ethnic disparities.
\end{abstract}

Keywords COVID-19 $\cdot$ SARS-CoV-2 $\cdot$ New York City $\cdot$ Risk factors $\cdot$ Racial/ethnic disparities $\cdot$ In-hospital mortality

\section{Introduction}

New York City (NYC) was an early United States (U.S.) epicenter of the coronavirus disease 2019 (COVID-19)

Dena Bushman and Alexander Davidson contributed equally to this work.

Dena Bushman

pgz3@cdc.gov

1 New York City Department of Health and Mental Hygiene, Queens, NY, USA

2 Epidemic Intelligence Service, Centers for Disease Control and Prevention, Atlanta, GA, USA pandemic [1]. By April 30, 2020, 174,501 confirmed COVID-19 cases, 47,779 hospitalizations, and 14,905 deaths occurred among NYC residents [2]. During this time, NYC public health messaging encouraged the public to stay home [3-7], possibly contributing to delays in obtaining emergency care $[8,9]$.

Among persons hospitalized with COVID-19 in the U.S., underlying conditions, including chronic kidney disease, cardiovascular disease, immunosuppression, chronic lung disease, neurologic disorders, diabetes, and obesity, have been identified as risk factors for mortality [10-13]. NYC-based COVID-19 in-hospital mortality studies have primarily used data from single hospital systems or focused on older persons, for whom elevated mortality rates have been observed [12-19]. However, many deaths in NYC were reported among 
persons aged 21-64 years. By April 30, 2020, such persons accounted for nearly $26 \%$ of COVID-19-related mortality [20]. In the U.S., COVID-19 diagnoses and hospitalizations have disproportionately impacted communities of color $[1,11$, 21-23]. While age-adjusted COVID-19 hospitalization and mortality rates in NYC have been higher among Hispanic/ Latino (1060 and 298 per 100,000, respectively) and nonHispanic Black (988 and 274 per 100,000, respectively) persons compared with non-Hispanic White (531 and 155 per 100,000, respectively) and non-Hispanic Asian (493 and 140 per 100,000, respectively) persons, higher in-hospital mortality risk among persons of color in NYC has not been previously reported [12, 13, 18, 19, 24].

We examined underlying conditions, stratified by race/ ethnicity and age, to assess risk factors associated with death among NYC residents aged 21-64 years hospitalized with COVID-19. These findings can inform clinicians and public health professionals in allocating resources and tailoring preventive measures during the COVID-19 pandemic.

\section{Methods}

\section{Data Sources}

Laboratories are required to electronically report SARS-CoV2 test results for NYC residents to the NY State Electronic Clinical Laboratory Reporting System [25]. To obtain hospitalization status, the NYC Department of Health and Mental Hygiene (DOHMH) matched patient identifiers from laboratory reports of confirmed COVID-19 cases with emergency department syndromic surveillance, the NY State Hospital Emergency Response Data System, regional health information organizations, NYC public hospitals, and DOHMH's death registry [1]. On March 26, 2020, the DOHMH requested remote access to electronic health records (EHRs) for patients hospitalized with COVID-19 in selected NY metropolitan area hospitals. Eighty hospitals and two temporary hospitals agreed, representing facilities where $89 \%$ of decedents between February 22 and April 9, 2020, were first hospitalized with COVID-19.

Investigators used a medical chart abstraction guidance document and an investigation form to abstract patients' demographics, substance use, occupation, past medical history, and clinical course. Substance use and occupation were inconsistently documented in EHRs and omitted from analyses. Presence of underlying conditions/symptoms was collected as "Indicated" when documented in EHRs, otherwise "Not indicated," because absence of documentation could not be assumed to mean absence of those conditions/symptoms. Categories of underlying conditions were created for (1) conditions prevalent among persons with COVID-19 [17, 21, 26] and (2) conditions commonly abstracted by investigations that did not fit existing categories. Data quality was supported by two independent investigators performing each abstraction. Study patients were matched [27] with the tuberculosis, HIV/AIDS, and hemoglobin A1c DOHMH registries [28] using standard key deterministic algorithms, and with the hepatitis $\mathrm{B}$ and $\mathrm{C}$ registries using a patient-level unique identifier. This activity was reviewed by the CDC and was conducted consistent with applicable federal law and CDC policy [1]. The DOHMH Institutional Review Board determined this activity to be non-research public health surveillance.

\section{Analytic Sample}

We conducted a matched case-control study of NYC residents aged 21-64 years who were reported to the DOHMH with nucleic acid amplification test-confirmed COVID-19 [29] during March 13-April 9, 2020, and were hospitalized $\geq 1$ time for reasons other than labor and delivery in facilities for which the DOHMH had EHR access. Sample size calculations for tests for two correlated proportions in a matched casecontrol design [30] were conducted using PASS 2019 (v19.0.3, NCSS, LLC, Kaysville, UT) (Appendix 1).

Among 15,097 eligible persons who met case-patient eligibility criteria described above, $1698(11.2 \%)$ died in an emergency department (ED) or hospital by April 13. Of these, $45(2.7 \%)$ were excluded because they died at an unknown location or outside of the hospital. Of the remaining 1653 eligible decedents, 350 were randomly selected, and medical chart abstraction was completed for 343 case-patients.

Control-patients met laboratory and hospitalization eligibility criteria described above, and were discharged alive from hospitalization(s). Matching was performed to reduce variance of effect estimates [31]. Control-patients were matched 2:1 to case-patients on age ( \pm 3 years, within the bounds of 21-64) and neighborhood of residence, as defined by the NYC United Hospital Fund [32]. Control-patients who left hospitals against medical advice or who were discharged to nursing homes, group homes, or step-down facilities were considered "discharged" and were included if they met the other inclusion criteria. Those discharged to hospice or still receiving inpatient hospital care at the time of medical chart abstraction were excluded and substituted with other matched control-patients. Among 13,399 eligible persons, medical chart abstractions were completed for 686 randomly selected, matched control-patients. Through a citywide death registry match, no known deaths among control-patients were documented as of July 1, 2020, when data collection was completed.

\section{Data Analysis}

To assess generalizability, given lack of EHR access to all NY metropolitan hospitals, we calculated relative risks (RRs) and 
95\% confidence intervals (CIs) to compare whether key demographics of case-patients differed significantly from other decedents who died in hospitals or EDs (including hospitals for which the DOHMH did not have EHR access) not selected for the study.

All case-patients with identical matching criteria and their matched controls were pooled, totaling 260 strata. We conducted conditional logistic regression analyses to evaluate associations between selected underlying conditions and odds of death and presented matched adjusted odds ratios (aORs) and 95\% CIs. All models included one condition of a priori interest and adjusted for sex (male, female) and two covariates thought to be potential confounders and significant in bivariate analyses: insurance status (private insurance, public insurance, uninsured) and marital status (married, not married).

Patient residence was assigned a neighborhood poverty level, defined as the percent of the population living in a given census tract whose household income was below the federal poverty level (FPL) per American Community Survey 2014-2018, with low poverty being $<10.0 \%$ below FPL, medium poverty $10.0-19.9 \%$ below FPL, high poverty $20.0-29.9 \%$ below FPL, and very high poverty $\geq 30.0 \%$ below FPL [33]. Obesity, a key exposure of interest, was considered in multiple ways. Body mass index (BMI) was calculated when height and weight were known. A five-level categorical variable for obesity was created based on National Institutes of Health $(\mathrm{NIH})$ criteria: "Underweight" (<18.5 kg/m²), "Normal weight" (18.5$\left.<25.0 \mathrm{~kg} / \mathrm{m}^{2}\right)$, "Overweight" $\left(25.0-<30.0 \mathrm{~kg} / \mathrm{m}^{2}\right)$, "Obese" $\left(30.0-<40.0 \mathrm{~kg} / \mathrm{m}^{2}\right)$, and "Morbidly obese" $\left(\geq 40.0 \mathrm{~kg} / \mathrm{m}^{2}\right)$ [34]. A two-level categorical obesity variable ("Indicated" vs. "Not indicated") was also created; obesity was "Indicated" if BMI was $\geq 30 \mathrm{~kg} / \mathrm{m}^{2}$ or BMI was unknown, but obesity was documented in the study patient's chart.

We described clinical characteristics of study patients, including presentation of symptoms, diagnoses of pneumonia and acute respiratory distress syndrome, use of respiratory support, initiation of dialysis, admission to the intensive care unit, and use of inpatient treatments. We evaluated combinations of comorbidities as risk factors for death by assessing the odds of death among patients with two specified underlying conditions. Conditions chosen for these analyses were prevalent (occurring among approximately $\geq 10 \%$ of case-patients) and significantly associated with death when adjusting for sex, marital status, and insurance status, and for which, directionality of causation is not always clear [35-39]. We analyzed combinations of conditions as four-level categorical variables, with patients having neither, one, or both conditions.

To assess racial/ethnic disparities in associations between selected underlying conditions and odds of death, we stratified analyses by the following racial/ethnic groups: non-Hispanic Asian (Asian), non-Hispanic Black (Black), Hispanic/Latino (Hispanic), nonHispanic White (White), and non-Hispanic (Other), which included non-Hispanic patients of other or multiple races. Patients of other races were included in regression models, but effect estimates for this group are not presented because of sparsity. We present one aOR for each racial/ethnic group per condition, comparing patients with the condition against patients for whom the condition was not indicated. To determine which conditions to include, we used the following criteria: any significant overall aOR with approximately $\geq 10 \%$ of case-patients having the condition, or, if not statistically significant, an overall aOR $\geq 1.5$ and $\geq 15 \%$ of case-patients having the condition. Because sparse data within some strata could contribute to underpowered analyses, we do not present stratified aORs for specific conditions that were part of grouped categories (e.g., hypertension as a subset of heart disease). To help explain racial/ethnic disparities in COVID-19 deaths in NYC, we assessed prevalence of underlying conditions by race/ethnicity among control-patients. Given minimal data collected during the pandemic on underlying condition prevalence among non-hospitalized COVID-19 patients, control-patients were the best available proxy for NYC's population to understand risk factors for inhospital mortality with COVID-19. Similarly, to assess differences in associations between selected conditions and odds of death among those aged $<50$ years and $\geq 50$ years, we calculated aORs restricted to each age group.

All analyses were conducted using SAS version 9.4 (Cary, $\mathrm{NC})$.

\section{Results}

Distributions of age, sex, borough of residence, and neighborhood-level poverty for case-patients $(\mathrm{n}=343)$ were similar to other decedents who died in hospitals or EDs (including hospitals for which the DOHMH did not have EHR access) but were not selected for the study (n $=1310$ ). Asian race/ethnicity was the only demographic characteristic that was significantly differentially distributed between case-patients $(9.1 \%)$ and decedents not selected for the study (5.0\%) (RR, 1.52 [95\% CI: $1.04,2.23])$.

Among study patients, the median age of both casepatients and control-patients was 56 years (range: 23-64 years) (Table 1); $95.2 \%$ of control-patients matched case-patients on age exactly ( \pm 0 years). Overall, patients were predominantly male $(65.5 \%)$, Hispanic 
Table 1 Matched odds ratios (mOR) comparing demographic information of study patients ( $\mathrm{N}=1029)$, by patient status- New York City, March 13April 9, 2020

\begin{tabular}{|c|c|c|c|c|}
\hline Characteristic & $\begin{array}{l}\text { Cases } \\
(\mathrm{n}=343)\end{array}$ & $\begin{array}{l}\text { Controls } \\
(\mathrm{n}=686)\end{array}$ & $\begin{array}{l}\text { Total } \\
(\mathrm{n}=1029)\end{array}$ & $\begin{array}{l}\mathrm{mOR} \\
(95 \% \mathrm{CI})^{1}\end{array}$ \\
\hline Median age (range) $)^{2}$ & $56(23-64)$ & $56(23-64)$ & $56(23-64)$ & \\
\hline \multicolumn{5}{|l|}{$\mathrm{Sex}-\mathrm{n}(\%)$} \\
\hline Female & $113(32.9)$ & $242(35.3)$ & $355(34.5)$ & $0.90(0.68,1.19)$ \\
\hline Male & $230(67.1)$ & $444(64.7)$ & $674(65.5)$ & Reference \\
\hline \multicolumn{5}{|l|}{ Race/ethnicity-n (\%) } \\
\hline Non-Hispanic Asian & $31(9.1)$ & $60(8.9)$ & $91(9.5)$ & $0.92(0.49,1.72)$ \\
\hline Non-Hispanic Black & 109 (31.9) & $187(27.7)$ & $296(29.1)$ & $1.13(0.69,1.86)$ \\
\hline Hispanic/Latino & $120(35.1)$ & $262(38.9)$ & $382(37.6)$ & $0.81(0.49,1.33)$ \\
\hline Non-Hispanic White & $48(14.0)$ & $98(14.5)$ & $146(14.4)$ & Reference \\
\hline Non-Hispanic Other ${ }^{3}$ & $34(9.9)$ & $67(9.9)$ & $101(12.9)$ & \\
\hline Unknown $^{4}$ & 1 & 12 & 13 & \\
\hline \multicolumn{5}{|l|}{ Borough of residence $^{2}-\mathrm{n}(\%)$} \\
\hline Bronx & $103(30.0)$ & $204(29.7)$ & $307(29.8)$ & \\
\hline Brooklyn & $76(22.2)$ & $150(21.9)$ & $226(22.0)$ & \\
\hline Manhattan & $21(6.1)$ & $45(6.6)$ & $66(6.4)$ & \\
\hline Queens & $127(37.0)$ & $255(37.2)$ & $382(37.1)$ & \\
\hline Staten Island & $16(4.7)$ & $32(4.7)$ & $48(4.7)$ & \\
\hline \multicolumn{5}{|c|}{ Proportion of population in census tract of residence living below the federal poverty level ${ }^{4}-\mathrm{n}(\%)$} \\
\hline$\geq 30.0 \%$ (very high poverty) & $82(24.1)$ & $145(21.3)$ & $227(22.2)$ & $1.58(0.93,2.68)$ \\
\hline $20.0 \%-<30.0 \%$ (high poverty) & $72(21.1)$ & $145(21.3)$ & $217(21.3)$ & $1.27(0.80,2.01)$ \\
\hline $10.0 \%-<20.0 \%$ (medium poverty) & $127(37.2)$ & $251(36.9)$ & $378(37.0)$ & $1.22(0.82,1.81)$ \\
\hline$<10.0 \%$ (low poverty) & $60(17.6)$ & $141(20.7)$ & $201(19.7)$ & Reference \\
\hline Unknown $^{4}$ & 2 & 6 & 8 & \\
\hline \multicolumn{5}{|l|}{ Marital status-n (\%) } \\
\hline Not married & $178(54.9)$ & $296(44.6)$ & $474(48.0)$ & $1.53(1.16,2.02)$ \\
\hline Married & $146(45.1)$ & $368(55.4)$ & $514(52.0)$ & Reference \\
\hline Unknown $^{4}$ & 19 & 22 & 41 & \\
\hline \multicolumn{5}{|l|}{ Insurance status - $\mathrm{n}(\%)$} \\
\hline Public & $202(58.9)$ & $308(44.9)$ & $510(49.6)$ & $1.22(0.82,1.83)$ \\
\hline Private & $92(26.8)$ & $287(41.8)$ & $379(36.8)$ & $0.58(0.38,0.90)$ \\
\hline Uninsured & $49(14.3)$ & $91(13.3)$ & $140(13.6)$ & Reference \\
\hline \multicolumn{5}{|l|}{ Pregnant ${ }^{6}$} \\
\hline Indicated & $0(0.0)$ & $3(1.2)$ & $3(0.8)$ & $--^{7}$ \\
\hline Not indicated & $113(100.0)$ & $239(98.8)$ & $352(99.2)$ & \\
\hline
\end{tabular}

\footnotetext{
${ }^{1}$ Bold-faced mORs indicate significance at alpha $=0.05$
}

${ }^{2}$ Odds ratios not produced for age or borough of residence, as study population was matched on age and United Hospital Fund neighborhood

${ }^{3}$ Non-Hispanic Other is included in the table and included in the regression model, but no OR is shown because the effect estimate has no meaningful interpretation

${ }^{4}$ Unknown numbers are shown in the table but were not included in the regression model

${ }^{5}$ American Community Survey 2014-2018 census tract data (defined according to 2010 boundaries)

${ }^{6}$ Restricted to female patients

${ }^{7}$ Undefined effect estimate

(37.6\%) or Black $(29.1 \%$ ), and residents of Queens $(37.1 \%)$ or the Bronx $(29.8 \%)$, and resided in medium poverty neighborhoods $(37.0 \%)$; these characteristics were similarly distributed among case-patients and control-patients, as expected from matching on residential neighborhood. However, case-patients were more likely to be unmarried (54.9\% vs. $44.6 \%)$ and less likely to have private health insurance $(26.8 \%$ vs. $41.8 \%)$. 
Table 2 Clinical information for study patients $(\mathrm{N}=1029)$, by patient status - New York City, March 13-April 9, 2020

\begin{tabular}{|c|c|c|c|}
\hline Clinical variables & $\begin{array}{l}\text { Cases }(\mathrm{n}=343) \\
\text { Number }(\%)\end{array}$ & $\begin{array}{l}\text { Controls }(\mathrm{n}=686) \\
\text { Number }(\%)\end{array}$ & $\begin{array}{l}\text { Total }(\mathrm{n}=1029) \\
\text { Number }(\%)\end{array}$ \\
\hline Symptomatic $^{1}$ & $342(99.7)$ & $685(99.9)$ & $1027(99.8)$ \\
\hline Median number of days from symptom onset to diagnosis in days (range) ${ }^{2}$ & $5(0-45)$ & $7(0-31)$ & $6(0-45)$ \\
\hline \multicolumn{4}{|l|}{ Symptoms } \\
\hline Fever & $300(87.7)$ & $629(91.8)$ & $929(90.5)$ \\
\hline Shortness of breath & $316(92.4)$ & $587(85.7)$ & $903(87.9)$ \\
\hline Cough & $273(79.8)$ & $615(89.9)$ & $888(86.5)$ \\
\hline Fatigue & $132(38.6)$ & $351(51.2)$ & $483(47.0)$ \\
\hline Myalgia & $121(35.4)$ & $351(51.2)$ & $472(46.0)$ \\
\hline Chills & $106(31.0)$ & $319(46.6)$ & $425(41.4)$ \\
\hline Diarrhea & $79(23.1)$ & $253(36.9)$ & $332(32.3)$ \\
\hline Nausea/vomiting & $73(21.4)$ & $220(32.1)$ & $293(28.5)$ \\
\hline Chest pain & $53(15.5)$ & $199(29.1)$ & $252(24.5)$ \\
\hline Anorexia & $46(13.4)$ & 189 (27.6) & 235 (22.9) \\
\hline Headache & $46(13.4)$ & $137(20.0)$ & $183(17.8)$ \\
\hline Sore throat & $36(10.5)$ & $122(17.8)$ & $158(15.4)$ \\
\hline Abdominal pain & $36(10.5)$ & $120(17.5)$ & $156(15.2)$ \\
\hline Altered mental status & $86(25.2)$ & $62(9.1)$ & $148(14.4)$ \\
\hline Nasal congestion & $30(8.8)$ & $100(14.6)$ & $130(12.7)$ \\
\hline Dizziness & $27(7.9)$ & $85(12.4)$ & $112(10.9)$ \\
\hline Sweating & $20(5.9)$ & $34(5.0)$ & $54(5.3)$ \\
\hline Loss of taste & $4(1.2)$ & $26(3.8)$ & $30(2.9)$ \\
\hline Loss of smell & $3(0.9)$ & $26(3.8)$ & $29(2.8)$ \\
\hline Bloody sputum & $9(2.6)$ & $17(2.5)$ & $26(2.5)$ \\
\hline Rigors & $4(1.2)$ & $12(1.8)$ & $16(1.6)$ \\
\hline \multicolumn{4}{|l|}{ Hospitalization course $^{3}$} \\
\hline \multicolumn{4}{|l|}{ Time to hospitalization ${ }^{4}$} \\
\hline $\begin{array}{l}\text { Median number of days from symptom onset to } \\
\text { first hospitalization admission (range) }\end{array}$ & $5(0-32)$ & $7(0-43)$ & $6(0-43)$ \\
\hline Pneumonia & $327(95.3)$ & $624(91.0)$ & $951(92.4)$ \\
\hline Acute respiratory distress syndrome & $202(58.9)$ & $66(9.6)$ & $268(26.0)$ \\
\hline \multicolumn{4}{|l|}{ Respiratory support } \\
\hline Supplemental oxygen & $296(86.3)$ & $575(83.8)$ & $871(84.6)$ \\
\hline Intubation/mechanical ventilation & $270(78.7)$ & $67(9.8)$ & $337(32.8)$ \\
\hline Continuous positive airway pressure/bilevel positive airway pressure & $68(19.8)$ & $38(5.5)$ & $103(10.0)$ \\
\hline Extracorporeal membrane oxygenation & $1(0.3)$ & $1(0.2)$ & $2(0.2)$ \\
\hline Dialysis initiated & $45(13.1)$ & $17(2.5)$ & $62(6.0)$ \\
\hline Intensive care unit admission & $229(66.7)$ & $102(14.9)$ & $331(32.2)$ \\
\hline Median length of stay in days (range) & $4(0-26)$ & $8.5(0-55)$ & $5(0-55)$ \\
\hline \multicolumn{4}{|l|}{ Inpatient treatment } \\
\hline Hydroxychloroquine & $302(88.1)$ & $559(81.5)$ & $861(83.7)$ \\
\hline Azithromycin & $294(85.7)$ & $495(72.2)$ & $789(76.7)$ \\
\hline Ascorbic acid & $75(21.9)$ & $158(23.0)$ & $233(22.6)$ \\
\hline Corticosteroids & $103(30.0)$ & $129(18.8)$ & $232(22.5)$ \\
\hline Tocilizumab $^{5}$ & $26(7.6)$ & $27(3.9)$ & $53(5.2)$ \\
\hline Oseltamivir & $24(7.0)$ & $35(5.1)$ & $59(5.7)$ \\
\hline Angiotensin-converting enzyme inhibitor & $9(2.6)$ & $15(2.2)$ & $24(2.3)$ \\
\hline Lopinavir/ritonavir & $7(2.0)$ & $10(1.4)$ & $17(1.7)$ \\
\hline Angiotensin II receptor blockers & $6(1.8)$ & $12(1.8)$ & $18(1.7)$ \\
\hline
\end{tabular}


Table 2 (continued)

\begin{tabular}{|c|c|c|c|}
\hline Clinical variables & $\begin{array}{l}\text { Cases }(\mathrm{n}=343) \\
\text { Number }(\%)\end{array}$ & $\begin{array}{l}\text { Controls }(\mathrm{n}=686) \\
\text { Number }(\%)\end{array}$ & $\begin{array}{l}\text { Total }(\mathrm{n}=1029) \\
\text { Number }(\%)\end{array}$ \\
\hline Remdesivir $^{5}$ & $5(1.5)$ & $9(1.3)$ & $14(1.4)$ \\
\hline Sarilumab $^{5}$ & $4(1.2)$ & $9(1.3)$ & $13(1.3)$ \\
\hline Chloroquine & $3(0.9)$ & $6(0.9)$ & $9(8.7)$ \\
\hline Convalescent plasma ${ }^{5}$ & $3(0.9)$ & $7(1.0)$ & $10(9.7)$ \\
\hline
\end{tabular}

${ }^{1}$ A patient was considered symptomatic if symptoms were noted in the EHR

${ }^{2}$ Based on 325 case-patients and 675 control-patients for whom onset date was available

${ }^{3}$ Some case-patients and control-patients were hospitalized more than once with COVID-19 within the study period. Hospitalization course data includes all hospitalizations with COVID-19 that occurred within the study period

${ }^{4}$ Time to hospitalization calculated as the number of days between symptom onset date and first hospitalization admission. This calculation is restricted to case-patients $(n=320)$ and control-patients $(n=676)$ with symptoms noted in the EHR, for whom symptom onset date was known, and for whom symptoms began before initial hospitalization

${ }^{5}$ Includes patients participating in clinical trials of this drug and who might have received placebo

\section{Hospitalization Course}

Most patients were known to be symptomatic prior to or during hospitalization (99.8\%); the most common symptoms were fever $(90.5 \%)$, shortness of breath $(87.9 \%)$, and cough $(86.5 \%)$ (Table 2). The median numbers of days from symptom onset to diagnosis and from symptom onset to first hospitalization were both 5 days for case-patients and 7 days for control-patients. Among case-patients, $95.3 \%$ and $58.9 \%$ had a diagnosis of pneumonia and acute respiratory distress syndrome, respectively, compared with $91.0 \%$ and $9.6 \%$ of controlpatients. Most case-patients (86.3\%) and control-patients $(83.8 \%)$ received supplemental oxygen, and $78.7 \%$ of case-patients and $9.8 \%$ of control-patients were intubated and placed on a mechanical ventilator. Among case-patients (90.1\%) and control-patients (96.5\%) who did not report being on dialysis at time of hospitalization, dialysis was initiated for $13.1 \%$ and $2.5 \%$ of case-patients and control-patients respectively. ICU admission occurred for $66.7 \%$ of case-patients and $14.9 \%$ of control-patients. The most common inpatient treatments administered to case-patients and controlpatients were hydroxychloroquine $(88.1 \%$ and $81.5 \%$, respectively), azithromycin $(85.7 \%$ and $72.2 \%)$, corticosteroids $(30.0 \%$ and $18.8 \%)$, and ascorbic acid $(21.9 \%$ and $23.0 \%)$.

\section{Underlying Conditions}

Most case-patients (96.5\%) and control-patients (86.4\%) had $\geq 1$ underlying condition (Table 3 ). After adjusting for sex, marital status, and insurance status, having $\geq 1$ selected underlying condition was strongly associated with death (aOR, 4.45 [95\% CI: 2.33, 8.49]).
Increased odds of death were observed for patients with diabetes (aOR, 2.09 [95\% CI: 1.56, 2.78]) and obesity (aOR,1.62 [95\% CI: 1.22, 2.14]), particularly morbid obesity (versus patients with normal BMI (aOR, 3.07 [95\% CI: 1.59, 5.90]). Heart disease was associated with an increased odds of death (aOR, 2.16 [95\% CI: $1.55,3.01]$ ); specifically, history of heart attack, congestive heart failure, coronary artery disease, and hypertension were each significantly associated with increased odds of death. Lung disease was associated with an increased odds of death (aOR, 1.52 [95\% CI: 1.08, 2.14]). Specifically, having chronic obstructive pulmonary disease/chronic bronchitis/emphysema was significantly associated with an increased odds of death (aOR, 2.40 [95\% CI: 1.39, 4.16]). Asthma was not significantly associated with death (aOR, 1.22 [95\% CI: 0.82, 1.81]). Patients with kidney disease had 2.51 (95\% CI: 1.72, 3.68) times the odds of death compared with those without kidney disease. Other underlying conditions significantly associated with death included cancer, neurologic or neurodevelopmental condition(s), mental health condition(s), anemia, and HIV/ AIDS.

\section{Combinations of Underlying Conditions}

Compared with having neither condition, having both heart disease and diabetes, obesity and diabetes, or hypertension and diabetes was significantly associated with an approximately threefold increased odds of death (Table 4). Patients with both heart disease and diabetes had 3.13 times $(95 \%$ CI: $2.11,4.62)$ the odds of death compared with those with neither heart disease nor diabetes. Patients with heart disease only had 1.97 times (95\% CI: 1.30, 2.97) the odds of death and patients 
Table 3 Matched adjusted odds ratios (aOR) of death with COVID-19 among study patients $(\mathrm{N}=1029)$ with specific underlying medical conditionsNew York City, March 13-April 9, 2020

\begin{tabular}{|c|c|c|c|}
\hline Condition $^{1}$ & $\begin{array}{l}\text { Cases } \\
(\mathrm{n}=343)\end{array}$ & $\begin{array}{l}\text { Controls } \\
(\mathrm{n}=686)\end{array}$ & $\begin{array}{l}\text { Matched aOR } \\
(95 \% \mathrm{CI})^{2}\end{array}$ \\
\hline \multicolumn{4}{|l|}{ Any underlying condition $-\mathrm{n}(\%)$} \\
\hline Indicated & $331(96.5)$ & $593(86.4)$ & $4.45(2.33,8.49)$ \\
\hline Not indicated & $12(3.5)$ & $93(13.6)$ & Reference \\
\hline \multicolumn{4}{|l|}{ Heart disease $\mathrm{e}^{3}-\mathrm{n}(\%)$} \\
\hline Indicated & $259(75.5)$ & $416(60.6)$ & $2.16(1.55,3.01)$ \\
\hline Not indicated & $84(24.5)$ & $270(39.4)$ & Reference \\
\hline \multicolumn{4}{|l|}{ Hypertension $-\mathrm{n}(\%)$} \\
\hline Indicated & $232(67.6)$ & $351(51.2)$ & $2.08(1.53,2.82)$ \\
\hline Not indicated & $111(32.4)$ & $335(48.8)$ & Reference \\
\hline \multicolumn{4}{|l|}{ Hyperlipidemia $-\mathrm{n}(\%)$} \\
\hline Indicated & $130(37.9)$ & $240(35.0)$ & $1.10(0.81,1.50)$ \\
\hline Not indicated & $213(62.1)$ & $446(65.0)$ & Reference \\
\hline \multicolumn{4}{|l|}{ Coronary artery disease $-\mathrm{n}(\%)$} \\
\hline Indicated & $58(16.9)$ & $64(9.3)$ & $1.66(1.10,2.51)$ \\
\hline Not indicated & $285(83.1)$ & $622(90.7)$ & Reference \\
\hline \multicolumn{4}{|l|}{ History of heart attack-n $(\%)$} \\
\hline Indicated & $35(10.2)$ & $21(3.1)$ & $3.41(1.87,6.23)$ \\
\hline Not indicated & $308(89.8)$ & $665(96.9)$ & Reference \\
\hline \multicolumn{4}{|l|}{ Congestive heart failure $-\mathrm{n}(\%)$} \\
\hline Indicated & $33(9.6)$ & $34(5.0)$ & $1.84(1.05,3.22)$ \\
\hline Not indicated & $310(90.4)$ & $652(95.0)$ & Reference \\
\hline \multicolumn{4}{|l|}{ Valvular heart disease $-\mathrm{n}(\%)$} \\
\hline Indicated & $19(5.5)$ & $16(2.3)$ & $1.85(0.88,3.87)$ \\
\hline Not indicated & $324(94.5)$ & $670(97.7)$ & Reference \\
\hline \multicolumn{4}{|l|}{ Congenital heart disease $-\mathrm{n}(\%)$} \\
\hline Indicated & $3(0.9)$ & $4(0.6)$ & $1.61(0.32,8.06)$ \\
\hline Not indicated & $340(99.1)$ & $682(99.4)$ & Reference \\
\hline \multicolumn{4}{|l|}{ Obesity $-\mathrm{n}(\%)$} \\
\hline Indicated & $209(60.9)$ & $346(50.4)$ & $1.62(1.22,2.14)$ \\
\hline Not indicated & $134(39.1)$ & $340(49.6)$ & Reference \\
\hline \multicolumn{4}{|l|}{ Body mass index category ${ }^{4}-\mathrm{n}(\%)$} \\
\hline Underweight $\left(<18.5 \mathrm{~kg} / \mathrm{m}^{2}\right)$ & $3(1.2)$ & $3(0.6)$ & $1.80(0.34,9.66)$ \\
\hline Normal weight $\left(18.5-<25.0 \mathrm{~kg} / \mathrm{m}^{2}\right)$ & $38(14.8)$ & $84(15.4)$ & Reference \\
\hline Overweight $\left(25.0-<30.0 \mathrm{~kg} / \mathrm{m}^{2}\right)$ & $69(26.8)$ & $195(35.7)$ & $0.88(0.52,1.50)$ \\
\hline Obese $\left(30.0-<40.0 \mathrm{~kg} / \mathrm{m}^{2}\right)$ & $98(38.1)$ & $217(39.7)$ & $1.19(0.72,1.96)$ \\
\hline Morbidly obese $\left(\geq 40.0 \mathrm{~kg} / \mathrm{m}^{2}\right)$ & $49(19.1)$ & $47(8.6)$ & $3.07(1.59,5.90)$ \\
\hline BMI unknown & 86 & 140 & \\
\hline \multicolumn{4}{|l|}{ Diabetes-n (\%) } \\
\hline Indicated & $192(56.0)$ & $250(36.4)$ & $2.09(1.56,2.78)$ \\
\hline Type 1 & $2(0.6)$ & $3(0.4)$ & \\
\hline Type 2 & $153(43.7)$ & $226(32.9)$ & \\
\hline Unknown & $37(10.8)$ & $21(3.1)$ & \\
\hline Not indicated & $151(44.0)$ & $250(63.6)$ & Reference \\
\hline \multicolumn{4}{|l|}{ Lung disease ${ }^{5}-\mathrm{n}(\%)$} \\
\hline Indicated & $90(26.2)$ & $131(19.1)$ & $1.52(1.08,2.14)$ \\
\hline Not indicated & $253(73.8)$ & $555(80.9)$ & Reference \\
\hline
\end{tabular}


Table 3 (continued)

\begin{tabular}{|c|c|c|c|}
\hline Condition $^{1}$ & $\begin{array}{l}\text { Cases } \\
(\mathrm{n}=343)\end{array}$ & $\begin{array}{l}\text { Controls } \\
(n=686)\end{array}$ & $\begin{array}{l}\text { Matched aOR } \\
(95 \% \mathrm{CI})^{2}\end{array}$ \\
\hline \multicolumn{4}{|l|}{ Asthma $-\mathrm{n}(\%)$} \\
\hline Indicated & $57(16.62)$ & $95(13.8)$ & $1.22(0.82,1.81)$ \\
\hline Not indicated & $286(83.38)$ & $591(86.2)$ & Reference \\
\hline \multicolumn{4}{|c|}{ Chronic obstructive pulmonary disease/bronchitis/emphysema $-\mathrm{n}(\%)$} \\
\hline Indicated & $34(9.9)$ & $29(4.2)$ & $2.40(1.39,4.16)$ \\
\hline Not indicated & $309(90.1)$ & $657(95.8)$ & Reference \\
\hline \multicolumn{4}{|l|}{ Cystic fibrosis - n $(\%)$} \\
\hline Indicated & 0 & 0 & $--^{6}$ \\
\hline Not indicated & $343(100.0)$ & $686(100.0)$ & \\
\hline \multicolumn{4}{|l|}{ Kidney disease $^{7}-\mathrm{n}(\%)$} \\
\hline Indicated & $79(23.0)$ & $71(10.3)$ & $2.51(1.72,3.68)$ \\
\hline Not indicated & $264(77.0)$ & $615(89.7)$ & Reference \\
\hline \multicolumn{4}{|l|}{ Renal failure on dialysis $-\mathrm{n}(\%)$} \\
\hline Indicated & $34(9.9)$ & $24(3.5)$ & $2.73(1.53,4.88)$ \\
\hline Not indicated & $309(90.1)$ & $662(96.5)$ & Reference \\
\hline \multicolumn{4}{|c|}{ Neurologic/neurodevelopmental condition ${ }^{8}-\mathrm{n}(\%)$} \\
\hline Indicated & $65(19.0)$ & $56(8.2)$ & $2.42(1.58,3.69)$ \\
\hline Not indicated & $278(81.0)$ & $630(91.8)$ & Reference \\
\hline \multicolumn{4}{|l|}{ Mental health condition ${ }^{9}-\mathrm{n}(\%)$} \\
\hline Indicated & $43(12.5)$ & $53(7.7)$ & $1.61(1.02,2.54)$ \\
\hline Not indicated & $300(87.5)$ & $633(92.3)$ & Reference \\
\hline \multicolumn{4}{|l|}{ Cancer-n $(\%)$} \\
\hline Indicated & $37(10.8)$ & $32(4.7)$ & $2.54(1.52,4.23)$ \\
\hline Diagnosed $<1$ year ago & $11(3.2)$ & $7(1.0)$ & \\
\hline Diagnosed 1 or more years ago & $26(7.6)$ & $25(3.6)$ & \\
\hline Not indicated & $306(89.2)$ & $654(95.3)$ & Reference \\
\hline \multicolumn{4}{|l|}{ Thyroid disorder $-\mathrm{n}(\%)$} \\
\hline Indicated & $33(9.6)$ & $47(6.9)$ & $1.38(0.84,2.27)$ \\
\hline Not indicated & $310(90.4)$ & $639(93.1)$ & Reference \\
\hline \multicolumn{4}{|l|}{ Anemia $-\mathrm{n}(\%)$} \\
\hline Indicated & $32(9.3)$ & $41(6.0)$ & $1.69(1.01,2.80)$ \\
\hline Not indicated & $311(90.7)$ & $645(94.0)$ & Reference \\
\hline \multicolumn{4}{|l|}{ Obstructive sleep apnea $-\mathrm{n}(\%)$} \\
\hline Indicated & $32(9.3)$ & $43(6.3)$ & $1.49(0.91,2.46)$ \\
\hline Not indicated & $311(90.7)$ & $643(93.7)$ & Reference \\
\hline \multicolumn{4}{|l|}{ Peripheral vascular disease $-\mathrm{n}(\%)$} \\
\hline Indicated & $29(8.5)$ & $32(4.7)$ & $1.59(0.89,2.84)$ \\
\hline Not indicated & $314(91.5)$ & $654(95.3)$ & Reference \\
\hline \multicolumn{4}{|l|}{ Liver disease ${ }^{10}-\mathrm{n}(\%)$} \\
\hline Indicated & $24(7.0)$ & $49(7.1)$ & $0.75(0.44,1.27)$ \\
\hline Not indicated & $319(93.0)$ & $637(92.9)$ & Reference \\
\hline \multicolumn{4}{|l|}{ Viral hepatitis - n $(\%)$} \\
\hline Indicated & $17(5.0)$ & $28(4.1)$ & $0.87(0.45,1.67)$ \\
\hline Not indicated & $326(95.0)$ & $658(95.9)$ & Reference \\
\hline \multicolumn{4}{|l|}{ Hepatitis $\mathrm{C}$ virus infection $-\mathrm{n}(\%)$} \\
\hline Indicated & $10(2.9)$ & $17(2.5)$ & $0.82(0.36,1.88)$ \\
\hline Not indicated & $333(97.1)$ & $669(97.5)$ & Reference \\
\hline
\end{tabular}


Table 3 (continued)

\begin{tabular}{|c|c|c|c|}
\hline Condition ${ }^{1}$ & $\begin{array}{l}\text { Cases } \\
(\mathrm{n}=343)\end{array}$ & $\begin{array}{l}\text { Controls } \\
(\mathrm{n}=686)\end{array}$ & $\begin{array}{l}\text { Matched aOR } \\
(95 \% \text { CI })^{2}\end{array}$ \\
\hline \multicolumn{4}{|c|}{ Hepatitis B virus infection- $\mathrm{n}(\%)$} \\
\hline Indicated & $9(2.6)$ & $11(1.6)$ & $1.28(0.48,3.40)$ \\
\hline Not indicated & $334(97.4)$ & $675(98.4)$ & Reference \\
\hline \multicolumn{4}{|l|}{ Cirrhosis } \\
\hline Indicated & $7(2.0)$ & $13(1.9)$ & $0.85(0.33,2.21)$ \\
\hline Not indicated & $336(98.0)$ & $673(98.1)$ & Reference \\
\hline \multicolumn{4}{|c|}{ Nonalcoholic fatty liver disease $-\mathrm{n}(\%)$} \\
\hline Indicated & $2(0.6)$ & $9(1.3)$ & $0.44(0.09,2.07)$ \\
\hline Not indicated & $341(99.4)$ & 677 (98.7) & Reference \\
\hline \multicolumn{4}{|l|}{ History of stroke $-\mathrm{n}(\%)$} \\
\hline Indicated & $23(6.7)$ & $27(3.9)$ & $1.43(0.78,2.62)$ \\
\hline Not indicated & $320(93.3)$ & $659(96.1)$ & Reference \\
\hline \multicolumn{4}{|c|}{ Autoimmune disease $-\mathrm{n}(\%)$} \\
\hline Indicated & $22(6.4)$ & $29(4.2)$ & $1.70(0.93,3.10)$ \\
\hline Not indicated & $321(93.6)$ & $657(95.8)$ & Reference \\
\hline \multicolumn{4}{|c|}{ Gastroesophageal reflux disorder $-\mathrm{n}(\%)$} \\
\hline Indicated & $19(5.5)$ & $57(8.3)$ & $0.65(0.36,1.16)$ \\
\hline Not indicated & $324(94.5)$ & $629(91.7)$ & Reference \\
\hline \multicolumn{4}{|l|}{ HIV/AIDS } \\
\hline Indicated & $15(4.4)$ & $13(1.9)$ & $2.42(1.05,5.59)$ \\
\hline AIDS & $3(0.9)$ & $3(0.4)$ & \\
\hline AIDS not indicated & $12(3.5)$ & $10(1.5)$ & \\
\hline Not indicated & $328(95.6)$ & $673(98.1)$ & Reference \\
\hline \multicolumn{4}{|c|}{ History of organ transplant $-\mathrm{n}(\%)$} \\
\hline Indicated & $8(2.3)$ & $6(0.9)$ & $2.97(0.95,9.27)$ \\
\hline Not indicated & 335 (97.7) & $680(99.1)$ & Reference \\
\hline \multicolumn{4}{|l|}{ Active tuberculosis- $\mathrm{n}(\%)$} \\
\hline Indicated & $1(0.3)$ & 0 & $--^{6}$ \\
\hline Not indicated & $342(99.7)$ & $686(100.0)$ & \\
\hline
\end{tabular}

${ }^{1}$ Conditions listed in descending order of prevalence among case-patients

${ }^{2}$ Adjusted for sex, marital status, and insurance status. Bold-faced aORs indicate significance at alpha $=0.05$

${ }^{3}$ Includes history of heart attack, congestive heart failure, coronary artery disease, hypertension, hyperlipidemia, congenital heart disease, valvular heart disease, and other chronic heart conditions (such as angina, arrhythmias, cardiomyopathy, and pulmonary hypertension)

${ }^{4}$ Defined according to National Institutes of Health criteria, weight $(\mathrm{kg}) /$ height $\left(\mathrm{m}^{2}\right)$

${ }^{5}$ Includes asthma, chronic obstructive pulmonary disease, chronic bronchitis, emphysema, cystic fibrosis, and other chronic lung conditions (such as pulmonary nodules, pulmonary fibrosis, and history of pulmonary emboli)

${ }^{6}$ Undefined effect estimate

${ }^{7}$ Includes chronic kidney conditions (such chronic kidney disease, glomerulonephritis, and polycystic kidney disease)

${ }^{8}$ Includes conditions such as seizure disorders, intellectual disability, dementia, history of traumatic brain injury, cerebral palsy, and neuropathy

${ }^{9}$ Includes conditions such as attention deficit hyperactivity disorder, depression, anxiety, bipolar disorder, schizophrenia, post-traumatic stress disorder, obsessive compulsive disorder, borderline personality disorder, and history of suicidal ideation

${ }^{10}$ Includes chronic liver conditions (such as cirrhosis, fatty liver disease, and chronic hepatitis, including viral hepatitis)

with diabetes only had 2.57 times (95\% CI: 1.33, 4.98) the odds of death compared with patients with neither condition. This pattern-patients with both conditions having increased odds of death compared with those having one or neither-was observed for all combinations considered. 
Table 4 Matched adjusted odds ratios (aOR) of death with COVID-19 for study patients $(\mathrm{N}=1029)$ with combinations of underlying medical conditions-New York City, March 13-April 9, 2020

\begin{tabular}{|c|c|c|c|}
\hline Condition combinations ${ }^{1}$ & $\begin{array}{l}\text { Cases } \\
(\mathrm{n}=343)\end{array}$ & $\begin{array}{l}\text { Controls } \\
(\mathrm{n}=686)\end{array}$ & $\begin{array}{l}\text { Matched aOR } 2 \\
(95 \% \text { CI })\end{array}$ \\
\hline \multicolumn{4}{|c|}{ Heart disease $^{3}$ and diabetes $-\mathrm{n}(\%)$} \\
\hline Both & $168(49.0)$ & $218(31.8)$ & $3.13(2.11,4.62$ \\
\hline Heart disease only & $91(26.5)$ & $198(28.9)$ & $1.97(1.30,2.97)$ \\
\hline Diabetes only & $24(7.0)$ & $32(4.7)$ & $2.57(1.33,4.98)$ \\
\hline Neither & $60(1$ & 238 & Reference \\
\hline \multicolumn{4}{|c|}{ Hypertension and diabetes $-\mathrm{n}(\%)$} \\
\hline Both & $156(45.5)$ & $195(28.4)$ & $2.98(2.06,4.30)$ \\
\hline Hypertension only & $76(22.2)$ & $156(22.7)$ & $1.88(1.25,2.81)$ \\
\hline Diabetes only & $36(10.5)$ & $55(8.0)$ & $2.13(1.25,3.63)$ \\
\hline Neither & $75(21.9)$ & $280(40.8)$ & Reference \\
\hline \multicolumn{4}{|c|}{ Hypertension and obesity $-\mathrm{n}(\%)$} \\
\hline Both & $151(44.0)$ & $200(29.2)$ & $2.90(1.94,4.32)$ \\
\hline Hypertension only & $81(23.6)$ & $151(22.0)$ & $2.06(1.35,3.15)$ \\
\hline Obesity only & $58(16.9)$ & $146(21.3)$ & $1.57(0.99,2.48)$ \\
\hline Neither & 53( & $189(27.6)$ & Reference \\
\hline \multicolumn{4}{|l|}{ Obesity and diabetes $-\mathrm{n}(\%)$} \\
\hline Both & $124(36.2)$ & $150(21.9)$ & $2.91(1.98,4.28$ \\
\hline Obesity only & $85(24.8)$ & $196(28.6)$ & $1.68(1.13,2.49$ \\
\hline Diabetes only & $68(1$ & $100(14.6)$ & $2.32(1.49,3.6$ \\
\hline Neither & $66(19.2)$ & 240 & Reference \\
\hline \multicolumn{4}{|c|}{ Hypertension and kidney disease ${ }^{4}-\mathrm{n}(\%)$} \\
\hline Both & $74(21.6)$ & $64(9.3)$ & $3.81(2.43,5.98$ \\
\hline Hypertension only & $158(46.1)$ & $287(41.8)$ & $1.81(1.31,2.5$ \\
\hline Kidney disease only & $5(1.5)$ & $7(1.0)$ & $2.10(0.54,8.18$ \\
\hline Neither & $106(30.9)$ & $328(47.8)$ & Reference \\
\hline \multicolumn{4}{|c|}{ Obesity and kidney disease ${ }^{4}-\mathrm{n}(\%)$} \\
\hline Both & $49(14.3)$ & $40(5.8)$ & $3.57(2.17,5.8$ \\
\hline Obesity only & $160(46.6)$ & $306(44.6)$ & $1.63(1.19,2.24)$ \\
\hline Kidney disease only & $30(8.7)$ & $31(4.5)$ & $2.84(1.56,5.16)$ \\
\hline Neither & $104(30.3)$ & $309(45.0)$ & Reference \\
\hline
\end{tabular}

${ }^{1}$ Conditions listed in descending order of prevalence among case-patients

${ }^{2}$ Adjusted for sex, marital status, and insurance status. Bold-faced aORs indicate significance at alpha $=0.05$

${ }^{3}$ Includes history of heart attack, congestive heart failure, coronary artery disease, hypertension, hyperlipidemia, congenital heart disease, valvular heart disease, and other chronic heart conditions (such as angina, arrhythmias, cardiomyopathy, and pulmonary hypertension)

${ }^{4}$ Includes chronic kidney conditions (such chronic kidney disease, glomerulonephritis, and polycystic kidney disease)

\section{Race/Ethnicity and Age Stratification}

The eight selected underlying conditions included in our stratified analysis had aOR point estimates $>1$ for death among all race/ethnicities, except lung disease among White patients (aOR, 0.85 [95\% CI: 0.36, 1.98]) (Table 5). Five of these
Table 5 Matched adjusted odds ratios (aOR) of death with COVID-19 among study patients $(\mathrm{N}=1029)$ with specific underlying medical conditions, stratified by race/ethnicity-New York City, March 13-April 9, 2020

\begin{tabular}{llll}
\hline Condition $^{1}$ & $\begin{array}{l}\text { Cases } \\
(\mathrm{n}=343)\end{array}$ & $\begin{array}{l}\text { Controls } \\
(\mathrm{n}=686)\end{array}$ & Matched aOR $(95 \% \mathrm{CI})^{2}$ \\
\hline
\end{tabular}

Any underlying condition $-\mathrm{n}(\%)$

Non-Hispanic Asian

$\begin{array}{llll}\text { Indicated } & 28(90.3) & 49(81.7) & 2.26(0.50,10.18) \\ \text { Not indicated } & 3(9.7) & 11(18.3) & \text { Reference }\end{array}$

Non-Hispanic Black

$\begin{array}{clll}\text { Indicated } & 108(99.1) & 179(95.7) & 4.03(0.46,35.44) \\ \text { Not indicated } & 1(0.9) & 8(4.3) & \text { Reference } \\ \text { Hispanic/Latino } & & & \\ \text { Indicated } & 114(95.0) & 214(81.7) & \mathbf{4 . 4 7}(\mathbf{1 . 8 4}, \mathbf{1 0 . 9 1}) \\ \text { Not indicated } & 6(5.0) & 48(18.3) & \text { Reference }\end{array}$

Non-Hispanic White

$\begin{array}{llll}\text { Indicated } & 48(100.0) & 85(86.7) & -^{3} \\ \text { Not indicated } & 0 & 13(13.3) & \text { Reference }\end{array}$

Heart disease ${ }^{4}-\mathrm{n}(\%)$

Non-Hispanic Asian

$$
\text { Indicated } \quad 25(80.6) \quad 35(58.3) \quad 2.50(0.84,7.43)
$$

Not indicated $6(19.4) \quad 25(41.7) \quad$ Reference

Non-Hispanic Black

Indicated $\quad 93(85.3) \quad 140(74.9) \quad \mathbf{2 . 3 6}(\mathbf{1 . 1 3}, \mathbf{4 . 9 4})$

Not indicated $16(14.7) \quad 47(25.1) \quad$ Reference

Hispanic/Latino

Indicated $\quad 79(65.8) \quad 133(50.8) \quad \mathbf{2 . 1 5}(\mathbf{1 . 3 1}, \mathbf{3 . 5 3})$

Not indicated $41(34.2) \quad 129(49.2) \quad$ Reference

Non-Hispanic White

$\begin{array}{llll}\text { Indicated } & 35(72.9) & 60(61.2) & 1.52(0.65,3.57) \\ \text { Not indicated } & 13(27.1) & 38(38.8) & \text { Reference }\end{array}$

Obesity-n (\%)

Non-Hispanic Asian

$\begin{array}{llll}\text { Indicated } & 11(35.5) & 13(21.7) & 2.09(0.71,6.10) \\ \text { Not indicated } & 20(64.5) & 47(78.3) & \text { Reference }\end{array}$

Non-Hispanic Black

Indicated $\quad 78(71.6) \quad 115(61.5) \quad 1.67(0.93,2.97)$

Not indicated $31(28.4) \quad 72(38.5) \quad$ Reference

Hispanic/Latino

Indicated $73(60.8)$

$133(50.8) \quad 1.73(\mathbf{1 . 1 0}, \mathbf{2 . 7 3})$

Not indicated 47 (39.2)

129 (49.2) Reference

Non-Hispanic White

$\begin{array}{llll}\text { Indicated } & 30(62.5) & 51(52.0) & 1.55(0.74,3.25) \\ \text { Not indicated } & 18(37.5) & 47(48.0) & \text { Reference }\end{array}$

Diabetes-n (\%)

Non-Hispanic Asian

\begin{tabular}{|c|c|c|c|}
\hline Indicated & $16(51.6)$ & $24(40.0)$ & $1.42(0.54,3.72)$ \\
\hline Not indicated & $15(48.4)$ & $36(60.0)$ & Reference \\
\hline \multicolumn{4}{|c|}{ Non-Hispanic Black } \\
\hline Indicated & $72(66.1)$ & $82(43.8)$ & $2.66(1.55,4.57)$ \\
\hline Not indicated & 37 (33.9) & $105(56.2)$ & Reference \\
\hline
\end{tabular}


Table 5 (continued)

\begin{tabular}{llll}
\hline Condition $^{1}$ & $\begin{array}{l}\text { Cases } \\
(\mathrm{n}=343)\end{array}$ & $\begin{array}{l}\text { Controls } \\
(\mathrm{n}=686)\end{array}$ & Matched aOR $(95 \% \mathrm{CI})^{2}$ \\
\hline
\end{tabular}

\section{Hispanic/Latino}

$60(50.0)$

$83(31.7)$

$1.97(1.24,3.12)$

Not indicated $60(50.0)$

$179(68.3)$

Reference

Non-Hispanic White

\begin{tabular}{|c|c|c|c|}
\hline Indicated & $24(50.0)$ & $31(21.6)$ & $2.07(0.96,4.49)$ \\
\hline Not indicated & $24(50.0)$ & $67(68.6)$ & Reference \\
\hline \multicolumn{4}{|c|}{ Lung disease ${ }^{5}-\mathrm{n}(\%)$} \\
\hline \multicolumn{4}{|c|}{ Non-Hispanic Asian } \\
\hline Indicated & $5(16.1)$ & $6(10.0)$ & $2.28(0.58,8.98)$ \\
\hline Not indicated & $26(83.9)$ & $54(90.0)$ & Reference \\
\hline \multicolumn{4}{|c|}{ Non-Hispanic Black } \\
\hline Indicated & $32(29.4)$ & $46(24.6)$ & $1.14(0.63,2.09)$ \\
\hline Not indicated & $77(70.6)$ & $141(75.4)$ & Reference \\
\hline \multicolumn{4}{|l|}{ Hispanic/Latino } \\
\hline Indicated & $30(25.0)$ & $44(16.8)$ & $1.97(1.10,3.54)$ \\
\hline Not indicated & $90(75.0)$ & $218(83.2)$ & Reference \\
\hline \multicolumn{4}{|c|}{ Non-Hispanic White } \\
\hline Indicated & $12(25.0)$ & $24(24.5)$ & $0.85(0.36,1.98)$ \\
\hline Not indicated & $36(75.0)$ & $74(75.5)$ & Reference \\
\hline \multicolumn{4}{|c|}{ Kidney disease ${ }^{6}-\mathrm{n}(\%)$} \\
\hline \multicolumn{4}{|c|}{ Non-Hispanic Asian } \\
\hline Indicated & $7(22.6)$ & $6(10.0)$ & $1.88(0.51,7.00)$ \\
\hline Not indicated & $24(87.4)$ & $54(90.0)$ & Reference \\
\hline \multicolumn{4}{|c|}{ Non-Hispanic Black } \\
\hline Indicated & $29(26.6)$ & $25(13.4)$ & $2.59(1.37,4.90)$ \\
\hline Not indicated & $90(73.4)$ & $162(86.6)$ & Reference \\
\hline \multicolumn{4}{|l|}{ Hispanic/Latino } \\
\hline Indicated & $30(25.0)$ & $26(9.9)$ & $3.31(1.75,6.27)$ \\
\hline Not indicated & $90(75.0)$ & $236(90.1)$ & Reference \\
\hline \multicolumn{4}{|c|}{ Non-Hispanic White } \\
\hline Indicated & $9(18.8)$ & $8(8.2)$ & $2.08(0.66,6.50)$ \\
\hline Not indicated & 39 (81.2) & $90(91.8)$ & Reference \\
\hline
\end{tabular}

Neurologic/neurodevelopmental condition ${ }^{7}-\mathrm{n}(\%)$

Non-Hispanic Asian

$\begin{array}{llll}\text { Indicated } & 2(6.5) & 4(6.7) & 1.20(0.19,7.60) \\ \text { Not indicated } & 29(93.5) & 56(93.3) & \text { Reference } \\ \text { Non-Hispanic Black } & & \\ \text { Indicated } & 24(22.0) & 19(10.2) & \mathbf{2 . 3 1}(\mathbf{1 . 0 9}, \mathbf{4 . 9 0}) \\ \text { Not indicated } & 85(78.0) & 168(89.8) & \text { Reference } \\ \text { Hispanic/Latino } & & & \\ \text { Indicated } & 13(10.8) & 17(6.5) & 1.70(0.75,3.84) \\ \text { Not indicated } & 107(89.2) & 245(93.5) & \text { Reference } \\ \text { Non-Hispanic White } & & \\ \text { Indicated } & 19(39.6) & 11(11.2) & \mathbf{3 . 9 4}(\mathbf{1 . 5 7 , 9 . 8 6}) \\ \text { Not indicated } & 29(60.4) & 87(88.8) & \text { Reference } \\ \text { Mental health condition }{ }^{8}-\mathrm{n}(\%) & \\ \text { Non-Hispanic Asian } & \end{array}$

Table 5 (continued)

\begin{tabular}{|c|c|c|c|}
\hline Condition $^{1}$ & $\begin{array}{l}\text { Cases } \\
(\mathrm{n}=343)\end{array}$ & $\begin{array}{l}\text { Controls } \\
(\mathrm{n}=686)\end{array}$ & Matched aOR $(95 \% \mathrm{CI})^{2}$ \\
\hline Indicated & $2(6.5)$ & $2(3.3)$ & $2.37(0.30,18.53)$ \\
\hline Not indicated & $29(93.5)$ & $58(96.7)$ & Reference \\
\hline \multicolumn{4}{|c|}{ Non-Hispanic Black } \\
\hline Indicated & $17(15.6)$ & $14(7.5)$ & $2.42(1.04,5.61)$ \\
\hline Not indicated & $92(84.4)$ & $173(92.5)$ & Reference \\
\hline \multicolumn{4}{|l|}{ Hispanic/Latino } \\
\hline Indicated & $9(7.5)$ & $14(5.3)$ & $1.57(0.64,3.87)$ \\
\hline Not indicated & $111(92.50)$ & $248(94.7)$ & Reference \\
\hline \multicolumn{4}{|c|}{ Non-Hispanic White } \\
\hline Indicated & $12(25.0)$ & $18(18.4)$ & $1.15(0.47,2.77)$ \\
\hline Not indicated & $36(75.0)$ & $80(8.6)$ & Reference \\
\hline \multicolumn{4}{|l|}{ Cancer- $\mathrm{n}(\%)$} \\
\hline \multicolumn{4}{|c|}{ Non-Hispanic Asian } \\
\hline Indicated & $4(12.9)$ & $4(6.7)$ & $2.36(0.52,10.66)$ \\
\hline Not indicated & $27(87.1)$ & $56(93.3)$ & Reference \\
\hline \multicolumn{4}{|c|}{ Non-Hispanic Black } \\
\hline Indicated & $9(8.3)$ & $10(5.3)$ & $1.40(0.54,3.65)$ \\
\hline Not indicated & $100(91.7)$ & $177(94.7)$ & Reference \\
\hline \multicolumn{4}{|l|}{ Hispanic/Latino } \\
\hline Indicated & $12(10.0)$ & $11(4.2)$ & $3.32(1.35,8.14)$ \\
\hline Not indicated & $108(90.0)$ & $251(95.8)$ & Reference \\
\hline \multicolumn{4}{|c|}{ Non-Hispanic White } \\
\hline Indicated & $7(14.6)$ & $3(3.1)$ & $4.43(1.04,18.79)$ \\
\hline Not indicated & $41(85.4)$ & $95(96.9)$ & Reference \\
\hline
\end{tabular}

${ }^{1}$ Any condition having a statistically significant aOR with approximately $10 \%$ prevalence among case-patients and any not statistically significant $\mathrm{aOR}$ of at least 1.5 and at least $15 \%$ prevalence among case-patients in unstratified analyses. Because of sparse data when stratifying, specific conditions that were part of grouped categories (e.g., heart disease, lung disease) were not presented on their own. Conditions listed in descending order by overall prevalence among case-patients

${ }^{2}$ Adjusted for patient sex, marital status, and insurance status. Bold-faced aORs indicate significance at alpha $=0.05$

${ }^{3}$ Undefined effect estimate

${ }^{4}$ Includes history of heart attack, congestive heart failure, coronary artery disease, hypertension, hyperlipidemia, congenital heart disease, valvular heart disease, and other chronic heart conditions (such as angina, arrhythmias, cardiomyopathy, and pulmonary hypertension)

${ }^{5}$ Includes asthma, chronic obstructive pulmonary disease, chronic bronchitis, emphysema, cystic fibrosis, and other chronic lung conditions (such as pulmonary nodules, pulmonary fibrosis, and history of pulmonary emboli)

${ }^{6}$ Includes chronic kidney conditions (such chronic kidney disease, glomerulonephritis, and polycystic kidney disease)

${ }^{7}$ Includes conditions such as seizure disorders, intellectual disability, dementia, history of traumatic brain injury, cerebral palsy, and neuropathy ${ }^{8}$ Includes conditions, such as attention deficit hyperactivity disorder, depression, anxiety, bipolar disorder, schizophrenia, post-traumatic stress disorder, obsessive compulsive disorder, borderline personality disorder, and history of suicidal ideation 
conditions were most prevalent among Black control-patients, including heart disease $(74.9 \%)$, obesity $(61.5 \%)$, diabetes $(43.8 \%)$, lung disease $(24.6 \%)$, and kidney disease $(13.4 \%)$, compared with control-patients of other races/ethnicities. Magnitudes of association for conditions in groups aged $<50$ and $\geq 50$ years were similar (Appendix 2).

\section{Discussion}

This case-control study assessed risk factors for death among approximately 1000 persons aged 21-64 years hospitalized with COVID-19 across 82 NY metropolitan area hospitals. We found having any of the underlying conditions examined was associated with an over 4-fold increase in odds of death.

Black and Hispanic populations in NYC are at higher risk of COVID-19 compared with other racial and ethnic groups [13, 18, 19, 24]. Additionally, COVID-19 mortality rates in NYC are highest among Black and Hispanic persons [20]. In our study, 67\% of casepatients were Black or Hispanic. Despite increased diagnosis and mortality rates, risk factors associated with greater in-hospital mortality among Black and Hispanic patients with COVID-19 have not been reported [13, 18, $19,24]$. We could not fully assess the association between race/ethnicity or poverty and in-hospital mortality because matching by neighborhood likely contributed to case-patients and control-patients having similar demographic and neighborhood-level characteristics. Race/ ethnicity was not independently associated with mortality among study patients, yet racial/ethnic disparities in deaths were observed in NYC. Notably, the prevalence of five conditions was highest among Black control-patients. A similar pattern has been observed among NYC residents aged $\geq 18$ years; the prevalence of hypertension, obesity, diabetes, and asthma among Black and Latino persons is higher compared with White and Asian persons [40]. Increased prevalence of conditions demonstrated to be associated with death likely contributed to the greater COVID-related mortality burden among Black and Latino persons in NYC.

In the U.S., White persons have a lower prevalence of diabetes and chronic kidney disease and lower rates of associated mortality compared with Black and Hispanic persons [41-43]. Racial/ethnic disparities associated with underlying condition prevalence and severe health outcomes can be explained by disparities in access to resources and opportunities, including health care [44, 45], housing [46], food [47], employment [44, 45], and education [48]. Further studies are needed to assess the associations between racial/ethnic disparities in these resources, SARS-CoV-2 infection, and severe outcomes. Additionally, research is needed to examine the associations between severe outcomes and (1) mitigation and severity of underlying conditions and (2) time to care.

Our findings may help guide the public health and medical communities' efforts to prevent COVID-19 using health communication campaigns encouraging vaccine uptake, social distancing, face coverings, and handwashing. While citywide COVID-19 cases, hospitalizations, and deaths decline, and vaccination rates rise, racial/ethnic disparities persist. As of June 2021, only $30 \%$ of Black and $39 \%$ of Hispanic NYC adult residents were fully vaccinated, compared with $47 \%$ of White and $67 \%$ of Asian NYC adult residents [49]. These observed racial/ethnic disparities, including those in underlying condition prevalence, underscore the importance of prioritizing and increasing COVID-19 prevention efforts in these communities. Such efforts may include provision of additional social services, increased health resources, and especially vaccination efforts for racial and ethnic minority groups, given the increased prevalence of underlying conditions that are risk factors for death with COVID-19 and the increased burden of overall mortality experienced by Black and Hispanic populations both during and after our study period [1].

Our study has limitations. First, the multiple EHR systems did not consistently capture information. Although known to be associated both with certain underlying conditions [50, 51] and death [52, 53], substance use and occupation could not be analyzed given incomplete data, which is a known limitation to EHR data [54]. Additionally, BMI was missing from $22 \%$ of study patients, potentially limiting our ability to distinguish risk among BMI categories. Second, clinicians may have had fewer opportunities to ascertain complete medical histories for case-patients before their deaths. By combining patients who did not have certain conditions with those for whom information was not captured, our observed effect sizes are likely more conservative than the true associations. Third, while controlpatients were discharged and not known to have died as of July 1, 2020, some might have been discharged to locations providing a higher level of care than they received prior to hospital admission for COVID-19 and/or might have experienced persistent or new symptoms related to COVID-19; such patients would be less likely to represent healthy controls, and observed associations between underlying conditions and death might be more conservative than true estimates. Fourth, stratified analyses by race/ethnicity were underpowered, and lack of observed significant associations should not be interpreted as no association. Fifth, we did not account for severity or control of underlying conditions, nor did 
we collect dosing information about inpatient treatments, so we cannot assess differences in association and death by these factors. Finally, generalizability of our results might be limited; our study was restricted to one city and hospitalized patients, and conducted early in the pandemic when the DOHMH had temporary surge staffing resources and remote EHR access, and when clinical management and treatment guidance was rapidly evolving. Despite resource constraints, which precluded us from extending the study period, findings nonetheless help explain why COVID-related health inequities persist within NYC.

Following the study period, COVID-19 mortality rates declined in NYC [20]. COVID-19 treatment guidelines [55] have evolved since the study period, which likely contributed to improved clinical decision-making and inpatient management. These circumstances reinforced our decision to only describe, and not conduct statistical tests on, the clinical characteristics of our study patients. Additionally, public health messaging in NYC shifted since the beginning of the pandemic, encouraging patients with severe symptoms to promptly seek care [56]. Obtaining comprehensive medical histories for hospitalized patients aged 21-64 years with COVID-19 is critical and may inform clinical decisionmaking since underlying conditions, particularly combinations of conditions, are risk factors for in-hospital mortality. Finally, observed racial/ethnic disparities in underlying condition prevalence, COVID-19 diagnosis, hospitalization, and mortality suggest the importance of increasing COVID-19 prevention efforts, especially among racial/ethnic minorities.

\section{Appendix 1. Sample size calculation details}

Based on prevalence estimates of two underlying conditions of interest, diabetes and obesity, in the general NYC adult population [56] and among patients diagnosed with COVID19 in NYC- and US-based studies [17-21], the expected prevalence of diabetes and of obesity for control-patients was $18 \%$ and $42 \%$, respectively. A sample size of 293 case-patients (for diabetes, yielding the larger minimum number of the two conditions) and two matched control-patients per case-patient, assuming the correlation coefficient for having diabetes between matched case- and control-patients was 0.20 , would achieve $80 \%$ power to detect a moderate effect size (i.e., odds ratio for diabetes prevalence of 1.70 versus the alternative of equal odds) using a chi-square test with $\alpha=0.05$. Thus, we aimed to include at least 300 case-patients and 600 matched control-patients.
Appendix 2. Matched adjusted odds ratios (aOR) of death with COVID-19 among study patients ( $N=$ 1029) with specific underlying medical conditions, stratified by age group-New York City, March 13April 9, 2020

\begin{tabular}{llll}
\hline Condition $^{1}$ & $\begin{array}{l}\text { Cases } \\
(\mathrm{n}=343)\end{array}$ & $\begin{array}{l}\text { Controls } \\
(\mathrm{n}=686)\end{array}$ & Matched aOR $(95 \% \mathrm{CI})^{2}$ \\
\end{tabular}

\begin{tabular}{|c|c|c|c|}
\hline \multicolumn{4}{|c|}{ Any underlying condition $-\mathrm{n}(\%)$} \\
\hline \multicolumn{4}{|l|}{$<50$ years } \\
\hline Indicated & $84(93.3)$ & $140(77.8)$ & $3.71(1.46,9.42)$ \\
\hline Not indicated & $6(6.7)$ & $40(22.2)$ & Reference \\
\hline \multicolumn{4}{|l|}{$\geq 50$ years } \\
\hline Indicated & $247(97.6)$ & $53(10.5)$ & $5.05(2.04,12.50)$ \\
\hline Not indicated & $6(2.37)$ & $453(89.5)$ & Reference \\
\hline \multicolumn{4}{|c|}{ Any heart disease ${ }^{3}-\mathrm{n}(\%)$} \\
\hline \multicolumn{4}{|l|}{$<50$ years } \\
\hline Indicated & $51(56.7)$ & $62(34.4)$ & $2.26(1.29,3.95)$ \\
\hline Not indicated & $39(43.3)$ & $118(65.6)$ & Reference \\
\hline \multicolumn{4}{|l|}{$\geq 50$ years } \\
\hline Indicated & $208(82.2)$ & $354(70.0)$ & $2.07(1.37,3.13)$ \\
\hline Not indicated & $45(17.8)$ & $152(30.0)$ & Reference \\
\hline \multicolumn{4}{|l|}{ Obesity-n (\%) } \\
\hline \multicolumn{4}{|l|}{$<50$ years } \\
\hline Indicated & $58(64.4)$ & $95(52.8)$ & $1.95(1.10,3.47)$ \\
\hline Not indicated & $32(35.6)$ & $85(47.2)$ & Reference \\
\hline \multicolumn{4}{|l|}{$\geq 50$ years } \\
\hline Indicated & $151(59.7)$ & $251(49.6)$ & $1.53(1.11,2.12)$ \\
\hline Not indicated & $102(40.3)$ & $255(50.4)$ & Reference \\
\hline \multicolumn{4}{|l|}{ Diabetes $-\mathrm{n}(\%)$} \\
\hline \multicolumn{4}{|l|}{$<50$ years } \\
\hline Indicated & $36(40.0)$ & $39(21.7)$ & $2.29(1.27,4.13)$ \\
\hline Not indicated & $54(60.0)$ & $141(78.3)$ & Reference \\
\hline \multicolumn{4}{|l|}{$\geq 50$ years } \\
\hline Indicated & $156(61.7)$ & $211(41.7)$ & $2.03(1.46,2.84)$ \\
\hline Not indicated & $97(38.3)$ & $295(58.3)$ & Reference \\
\hline \multicolumn{4}{|c|}{ Any lung disease ${ }^{4}-\mathrm{n}(\%)$} \\
\hline \multicolumn{4}{|l|}{$<50$ years } \\
\hline Indicated & $22(24.4)$ & $40(22.2)$ & $1.06(0.54,2.11)$ \\
\hline Not indicated & $68(75.6)$ & $140(77.8)$ & Reference \\
\hline \multicolumn{4}{|l|}{$\geq 50$ years } \\
\hline Indicated & $68(26.9)$ & $91(18.0)$ & $1.73(1.16,2.58)$ \\
\hline Not indicated & $185(73.1)$ & $415(82.0)$ & Reference \\
\hline \multicolumn{4}{|c|}{ Any kidney disease ${ }^{5}-\mathrm{n}(\%)$} \\
\hline \multicolumn{4}{|l|}{$<50$ years } \\
\hline Indicated & $14(15.6)$ & $9(5.0)$ & $3.05(1.18,7.84)$ \\
\hline Not indicated & $76(84.4)$ & $171(95.0)$ & Reference \\
\hline \multicolumn{4}{|l|}{$\geq 50$ years } \\
\hline Indicated & $65(25.7)$ & $62(12.3)$ & $2.38(1.55,3.58)$ \\
\hline
\end{tabular}


Not indicated 188 (74.3) 444 (87.7) Reference

Any neurologic/neurodevelopmental condition ${ }^{6}-\mathrm{n}(\%)$

$<50$ years

$\begin{array}{llll}\text { Indicated } & 17(18.9) & 12(6.7) & \mathbf{3 . 5 5}(\mathbf{1 . 4 0 , 8 . 9 8}) \\ \text { Not indicated } & 73(81.1) & 168(93.3) & \text { Reference }\end{array}$

$\geq 50$ years

Indicated $\quad 48(19.0) \quad 44(8.7) \quad \mathbf{2 . 1 6}(\mathbf{1 . 3 3}, \mathbf{3 . 4 9})$

Not indicated $205(81.0) \quad 462(91.3) \quad$ Reference

Any mental health diagnosis ${ }^{7}-\mathrm{n}(\%)$

$<50$ years

Indicated $10(11.1) \quad 10(5.6) \quad 1.84(0.69,4.86)$

Not indicated $\quad 80(88.9) \quad 170(94.4) \quad$ Reference

$\geq 50$ years

Indicated $\quad 33(13.0) \quad 43(8.5) \quad 1.55(0.92,2.61)$

Not indicated $220(87.0) \quad 463(91.5) \quad$ Reference

Cancer

$\begin{array}{llll}<50 \text { years } & & & \\ \text { Indicated } & 5(5.6) & 2(1.1) & 4.16(0.74,23.33) \\ \text { Not indicated } & 85(94.4) & 178(98.9) & \text { Reference } \\ \geq 50 \text { years } & & & \\ \text { Indicated } & 32(12.7) & 30(5.9) & \mathbf{2 . 3 4}(\mathbf{1 . 3 6}, \mathbf{4 . 0 3}) \\ \text { Not indicated } & 221(87.3) & 476(94.1) & \text { Reference }\end{array}$

${ }^{1}$ Any condition having a statistically significant aOR with approximately $10 \%$ prevalence among case-patients and any not statistically significant $\mathrm{aOR}$ of at least 1.5 and at least $15 \%$ prevalence among case-patients in unstratified analyses. Because of sparse data when stratifying, specific conditions that were part of grouped categories (e.g., heart disease, lung disease) were not presented on their own. Conditions listed in descending order by overall prevalence among case-patients

${ }^{2}$ Adjusted for patient sex, marital status, and insurance status. Bold-faced aORs indicate significance at alpha $=0.05$

${ }^{3}$ Includes history of heart attack, congestive heart failure, coronary artery disease, hypertension, hyperlipidemia, congenital heart disease, valvular heart disease, and other chronic heart conditions (such as angina, arrhythmias, cardiomyopathy, and pulmonary hypertension)

${ }^{4}$ Includes asthma, chronic obstructive pulmonary disease, chronic bronchitis, emphysema, cystic fibrosis, and other chronic lung conditions (such as pulmonary nodules, pulmonary fibrosis, and history of pulmonary emboli)

${ }^{5}$ Includes chronic kidney conditions (such as chronic kidney disease, glomerulonephritis, and polycystic kidney disease)

${ }^{6}$ Includes conditions such as seizure disorders, intellectual disability, dementia, history of traumatic brain injury, cerebral palsy, and neuropathy

${ }^{7}$ Includes conditions, such as attention deficit hyperactivity disorder, depression, anxiety, bipolar disorder, schizophrenia, post-traumatic stress disorder, obsessive compulsive disorder, borderline personality disorder, and history of suicidal ideation

\section{Appendix 3. Sample code looking at interaction analyses by race/ethnicity}

proc logistic data=analysis;

class pc_exists (ref="No") raceeth (ref="White") gender (ref="FEMALE") ins (ref="Private") marital (ref="Married") / param=ref; model outbreak_inv_status (event="Case") = pc_exists raceeth

pc_exists*raceeth gender ins marital;

strata strata;

oddsratio pc_exists / at(raceeth="Asian");

oddsratio pc_exists / at(raceeth="Black");

oddsratio pc_exists / at(raceeth="Hispanic");

oddsratio pc_exists / at(raceeth="White");

run;

/*variable names*/

*pc_exists: presence of any underlying condition;

*raceeth: patient race/ethnicity;

*gender: patient sex;

*ins: patient insurance status;

*marital: patient marital status;

*outbreak_inv_status: case vs. control status;

*strata: pooled strata identifier;

Acknowledgements Jennifer Baumgartner, Katelynn Devinney, Meredith Eddy, Annie Fine, Kevin Guerra, Emily McGibbon, Kenya Murray, Stephanie Ngai, Marc Paladini, Hilary Parton, Kathleen Reilly, Corinne Thompson, Amanda Wahnich, Incident Command System Surveillance and Epidemiology Emergency Response Group, New York City Department of Health and Mental Hygiene; Mary Huynh, Gretchen van Wye, Wen Hui Li, Bureau of Vital Statistics, New York City Department of Health and Mental Hygiene; Anna Blackstock, National Center for Emerging and Zoonotic Infectious Diseases, Centers for Disease Control and Prevention; Byron Robinson, Center for Surveillance, Epidemiology, and Laboratory Services, Centers for Disease Control and Prevention; Rebecca Kahn, Center for Communicable Disease Dynamics, Harvard T.H. Chan School of Public Health

New York City Fatal Case-Control Study Team Milhardi Adonis, Mike Antwi, MD, MPH; Tracey Assanah, MPH; Dominique Balan, MPH; Vennus Ballen, MD; Nicole Burton PhD, MPH; Jonathan Chan, BA; Ashley Cintron, MPH; Marie Dorsinville RN, MPH; Daniel Eiras, MD, MPH; Eva Fabian, MPH; Anita Geevarughese, MD, MPH; Tingting Gu-Templin, MD; Liat Hotz; Maura Lash, RN, MPH; Ellen Lee, MD; Christina Ng, RN, BSN; Rachel Paneth-Pollak, MD, MPH; Emma Ruderman, MD; Amita Toprani, MD; Isatu Toure-Kaba; Ann Winters, MD

Availability of Data and Material Line-level patient data are not publicly available in accordance with patient confidentiality and privacy laws. Public data are available from: https://www1.nyc.gov/site/doh/covid/ covid-19-data.page

Code Availability This analysis used standard conditional logistic regression techniques and did not require custom code. Sample code is provided in Appendix 3.

\section{Declarations}

Ethics Approval This activity was reviewed by the CDC and was conducted consistent with applicable federal law and CDC policy. The 
DOHMH Institutional Review Board determined this activity to be nonresearch public health surveillance.

\section{Consent to Participate Not applicable}

Consent for Publication Not applicable

Conflict of Interest Not applicable

Disclaimer The findings and conclusions in the article are those of the authors and do not necessarily represent the official position of the U.S. Centers for Disease Control and Prevention (CDC).

Open Access This article is licensed under a Creative Commons Attribution 4.0 International License, which permits use, sharing, adaptation, distribution and reproduction in any medium or format, as long as you give appropriate credit to the original author(s) and the source, provide a link to the Creative Commons licence, and indicate if changes were made. The images or other third party material in this article are included in the article's Creative Commons licence, unless indicated otherwise in a credit line to the material. If material is not included in the article's Creative Commons licence and your intended use is not permitted by statutory regulation or exceeds the permitted use, you will need to obtain permission directly from the copyright holder. To view a copy of this licence, visit http://creativecommons.org/licenses/by/4.0/.

\section{References}

1. Thompson CN, Baumgartner J, Pichardo C, Toro B, Li L, Arciuolo R, et al. COVID-19 outbreak - New York City, February 29-June 1, 2020. MMWR Morb Mortal Wkly Rep. 2020;69(46):1725-9.

2. NYC Coronavirus Disease 2019 (COVID-19) Data. Data by day. https:// github.com/nychealth/coronavirus-data Accessed January 3, 2021.

3. @ nycHealthy "If you are sick, STAY HOME. If you do not feel better in 3 to 4 days, consult with your health care provider. Try to call, text, telemedicine or use your patient portal to contact your physician." https://twitter.com/nycHealthy/status/ 1239373468392665091 March 15, 2020 Tweet.

4. New York City Department of Education. Coronavirus Update for Families March 10, 2020. https:/infohub.nyced.org/docs/defaultsource/default-document-library/coronavirus-update-forfamilies_march-10-2020-(002).pdf Accessed February 9, 2021.

5. New York State Department of Health. Protect yourself from COVID-19 and stop the spread of germs. 3/20. https://www1.nyc. gov/assets/acs/pdf/covid19/covidprotectyourselfposter.pdf Accessed February 9, 2021.

6. Notify NYC. Notification issued 03-17-2020: "Do your part NYC, stay home! Visit the ER only if you are severely ill, otherwise you jeopardize the lives of others who are ill." https://a858nycnotify.nyc.gov/notifynyc?AspxAutoDetectCookieSupport=1.

7. New York City Health Commissioner COVID-19 Public Service Announcement March 23, 2020. https://www.youtube.com/ watch? $=$ SJE7gcxPJE0

8. Hartnett KP, Kite-Powell A, DeVies J, Coletta MA, Boehmer TK, Adjemian J, et al. Impact of the COVID-19 Pandemic on Emergency Department Visits - United States, January 1, 2019May 30, 2020. MMWR Morb Mortal Wkly Rep. 2020;69(23): 699-704.

9. New York City Department of H, Mental Hygiene C-RT. Preliminary estimate of excess mortality during the COVID-19 outbreak - New York City, March 11-May 2, 2020. MMWR Morb Mortal Wkly Rep 2020;69(19):603-605.

10. Fried MW, Crawford JM, Mospan AR, et al. Patient characteristics and outcomes of 11,721 patients with COVID19 hospitalized across the United States. Clin Infect Dis. 2020.

11. Kim L, Garg S, O'Halloran A, et al. Risk factors for intensive care unit admission and in-hospital mortality among hospitalized adults identified through the U.S. Coronavirus Disease 2019 (COVID19)-Associated Hospitalization Surveillance Network (COVIDNET). Clin Infect Dis. 2020.

12. Kalyanaraman Marcello R, Dolle J, Grami S, Adule R, Li Z, Tatem $\mathrm{K}$, et al. Characteristics and outcomes of COVID-19 patients in New York City's public hospital system. PLoS One. 2020;15(12): e0243027.

13. Petrilli CM, Jones SA, Yang J, et al. Factors associated with hospital admission and critical illness among 5279 people with coronavirus disease 2019 in New York City: prospective cohort study. bmj. 2020;369.

14. Paranjpe I, Russak AJ, De Freitas JK, et al. Retrospective cohort study of clinical characteristics of 2199 hospitalised patients with COVID-19 in New York City. BMJ Open. 2020;10(11):e040736.

15. Gayam V, Chobufo MD, Merghani MA, Lamichhane S, Garlapati PR, Adler MK. Clinical characteristics and predictors of mortality in African-Americans with COVID-19 from an inner-city community teaching hospital in New York. J Med Virol. 2021;93(2):812-9.

16. Richardson S, Hirsch JS, Narasimhan M, Crawford JM, McGinn T, Davidson KW, et al. Presenting characteristics, comorbidities, and outcomes among 5700 patients hospitalized with COVID-19 in the New York City area. JAMA. 2020;323:2052-9.

17. Lighter J, Phillips M, Hochman S, Sterling S, Johnson D, Francois $\mathrm{F}$, et al. Obesity in patients younger than 60 years is a risk factor for COVID-19 hospital admission. Clin Infect Dis. 2020;71(15):896-7.

18. Kabarriti R, Brodin NP, Maron MI, Guha C, Kalnicki S, Garg MK, et al. Association of race and ethnicity with comorbidities and survival among patients with COVID-19 at an urban medical center in New York. JAMA Netw Open. 2020;3(9):e2019795.

19. Wang Z, Zheutlin A, Kao YH, Ayers K, Gross S, Kovatch P, et al. Hospitalised COVID-19 patients of the Mount Sinai Health System: a retrospective observational study using the electronic medical records. BMJ Open. 2020;10(10):e040441.

20. New York City Department of Health and Mental Hygiene. COVID-19: Totals Data: https://www1.nyc.gov/site/doh/covid/ covid-19-data-totals.page Accessed December 31, 2020.

21. Garg S, Kim L, Whitaker M, O'Halloran A, Cummings C, Holstein $\mathrm{R}$, et al. Hospitalization rates and characteristics of patients hospitalized with laboratory-confirmed coronavirus disease 2019 COVID-NET, 14 states, March 1-30, 2020. MMWR Morb Mortal Wkly Rep. 2020;69(15):458-64.

22. Wortham JM, Lee JT, Althomsons S, Latash J, Davidson A, Guerra $\mathrm{K}$, et al. Characteristics of persons who died with COVID-19 United States, February 12-May 18, 2020. MMWR Morb Mortal Wkly Rep. 2020;69(28):923-9.

23. Rosenthal N, Cao Z, Gundrum J, Sianis J, Safo S. Risk factors associated with in-hospital mortality in a US national sample of patients with COVID-19. JAMA Netw Open. 2020;3(12): e2029058.

24. Ogedegbe G, Ravenell J, Adhikari S, Butler M, Cook T, Francois F, et al. Assessment of racial/ethnic disparities in hospitalization and mortality in patients with COVID-19 in New York City. JAMA Netw Open. 2020;3(12):e2026881.

25. New York State Department of Health. Health advisory: reporting requirements for all laboratory results for SARS-CoV-2 IaM, antigen, and serological tests (including "rapid" tests) and ensuring complete reporting of patient demographics. https:// coronavirus.health.ny.gov/system/files/documents/2020/04/ 
doh_covid19_reportingtestresults_rev_043020.pdf. Accessed Februrary 16, 2021. .

26. Team CC-R. Preliminary estimates of the prevalence of selected underlying health conditions among patients with coronavirus disease 2019 - United States, February 12-March 28, 2020. MMWR Morb Mortal Wkly Rep. 2020;69(13):382-6.

27. Drobnik A, Pinchoff J, Bushnell G, Ly S, Yuan J, Varma JK, et al. Matching HIV, tuberculosis, viral hepatitis, and sexually transmitted diseases surveillance data, 2000-2010: identification of infectious disease syndemics in New York City. J Public Health Manag Pract. 2014;20(5):506-12.

28. Chamany S, Silver LD, Bassett MT, et al. Tracking diabetes: New York City's A1C Registry. Milbank Q. 2009;87(3):547-70.

29. Coronavirus Disease 2019 (COVID-19) 2020 Interim Case Definition, Approved April 5, 2020 https:/wwwn.cdc.gov/nndss/ conditions/coronavirus-disease-2019-covid-19/case-definition/ 2020/ Accessed January 25, 2021.

30. Dupont WD. Power calculations for matched case-control studies. Biometrics. 1988;44(4):1157-68.

31. Mansournia MA, Hernan MA, Greenland S. Matched designs and causal diagrams. Int J Epidemiol. 2013;42(3):860-9.

32. United Hospital Fund Codes. https://www1.nyc.gov/assets/doh/ downloads/pdf/ah/zipcodetable.pdf Accessed January 3, 2021.

33. Toprani A HJ. Selecting and applying a standard area-based socioeconomic status measure for public health data: analysis for New York City. New York City Department of Health and Mental Hygiene: Epi Research Report. 2013:1e11.

34. Weir CB, Jan A. BMI classification percentile and cut off points. In: StatPearls. Treasure Island (FL)2020.

35. Ferrannini E, Cushman WC. Diabetes and hypertension: the bad companions. Lancet. 2012;380(9841):601-10.

36. Al-Goblan AS, Al-Alfi MA, Khan MZ. Mechanism linking diabetes mellitus and obesity. Diabetes Metab Syndr Obes. 2014;7:58791.

37. Shah S, Thakar CV. Diabetes/kidney/heart disease. Cardiol Clin. 2019;37(3):ix-x.

38. Hall JE. do Carmo JM, da Silva AA, Wang Z, Hall ME. Obesity, kidney dysfunction and hypertension: mechanistic links. Nat Rev Nephrol. 2019;15(6):367-85.

39. Clodi M, Saly C, Hoppichler F, Resl M, Steinwender C, Eber B. [Diabetes mellitus, coronary artery disease and heart disease]. Wien Klin Wochenschr. 2016;128 Suppl 2:S212-215.

40. New York City Department of Health and Mental Hygiene. Community Health Survey 2014-2018. https://www1.nyc.gov/ site/doh/data/data-sets/community-health-survey-public-use-data. page. Accessed 13 May 2020.

41. Rosenstock S, Whitman S, West JF, Balkin M. Racial disparities in diabetes mortality in the 50 most populous US cities. $J$ Urban Health. 2014;91(5):873-85.

42. Assari S. Racial disparities in chronic kidney diseases in the United States; a pressing public health challenge with social, behavioral and medical causes. $J$ Nephropharmacol. 2016;5(1):4-6.
43. Centers for Disease Control and Prevention. Chronic Kidney Disease Surveillance System-United States. http://www.cdc. gov/ckd Accessed January 22, 2021.

44. Bleich SN, Findling MG, Casey LS, Blendon RJ, Benson JM, SteelFisher GK, et al. Discrimination in the United States: experiences of black Americans. Health Serv Res. 2019;54(Suppl 2): 1399-408.

45. Findling MG, Bleich SN, Casey LS, Blendon RJ, Benson JM, Sayde JM, et al. Discrimination in the United States: experiences of Latinos. Health Serv Res. 2019;54(Suppl 2):1409-18.

46. Economic Policy Institute. Black workers face two of the most lethal preexisting conditions for coronavirus - racism and economic inequality [online]. 2020 [cited 2020 Jun 28].Accessed December 16, 2020: https://www.epi.org/publication/blackworkers-covid/external

47. Odoms-Young A, Bruce MA. Examining the impact of structural racism on food insecurity: implications for addressing racial/ethnic disparities. Fam Community Health. 2018;41 Suppl 2 Suppl, Food Insecurity and Obesity:S3-S6.

48. Assari S. Race, education attainment, and happiness in the United States. Int J Epidemiol Res. 2019;6(2):76-82.

49. New York City Department of Health and Mental Hygiene. COVID-19: vaccine data: https://www1.nyc.gov/site/doh/covid/ covid-19-data-vaccines.page Accessed June 1, 2021.

50. Bahorik AL, Satre DD, Kline-Simon AH, Weisner CM, Campbell CI. Alcohol, cannabis, and opioid use disorders, and disease burden in an integrated health care system. J Addiction Med. 2017;11(1):39. https://doi.org/10.1097/ADM.0000000000000260.

51. Dembe AE, Yao X. Chronic disease risks from exposure to longhour work schedules over a 32-year period. J Occup Environ Med. 2016;58(9):861-7.

52. Glei DA, Preston SH. Estimating the impact of drug use on US mortality, 1999-2016. PLoS One. 2020;15(1):e0226732.

53. Census of fatal occupational injuries summary, 2019. U.S Bureau of Labor Statistics. Accessed December 16, 2020: https:// www.bls.gov/news.release/cfoi.nr0.htm.

54. Kohane IS, Aronow BJ, Avillach P, Beaulieu-Jones BK, Bellazzi $\mathrm{R}$, Bradford RL, et al. What every reader should know about studies using electronic health record data but may be afraid to ask. $J$ Med Internet Res. 2021;23.

55. Bhimraj A, Morgan RL, Shumaker AH, et al. Infectious Diseases Society of America guidelines on the treatment and management of patients with COVID-19. Clin Infect Dis. 2020.

56. New York City Department of Health and Mental Hygiene. 2020 Health Advisory \#17: Advise Patients When to Seek Essential Care and Plan to Expand In-Person Patient Services https://www1.nyc. gov/assets/doh/downloads/pdf/han/advisory/2020/covid-19. restoring-health-care.pdf Accessed January 14, 2021.

Publisher's Note Springer Nature remains neutral with regard to jurisdictional claims in published maps and institutional affiliations. 